\title{
Mon corps et moi: quel couple!
}

\section{Piet van Spijk}

Dr méd., Dr phil., spécialiste en médecine interne générale, membre de la FMH, président du Forum Medizin und Philosophie

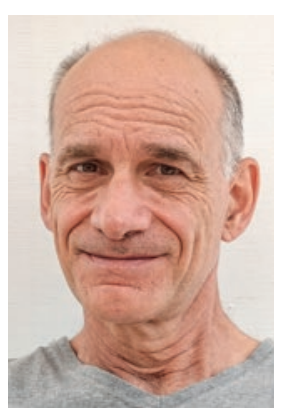

«'ai poussé mon corps au-delà de ses limites, il a fini par se rebeller, par m’asséner 'Ça suffit!' et par me jeter au lit.» Ou: «Au fil des ans, j’ai appris à écouter mon corps et à lui faire confiance.»

Ce genre d'affirmations m'interpelle. Je n'en reviens pas de tout ce que mon corps dit et fait. Bien sûr, il est réconfortant de savoir que l'on n'est pas seul dans la vie, mais en bonne compagnie. Il n'y a donc rien à redire au fait que mon corps et moi formons un couple, même si les deux partenaires, comme c'est le cas dans de nombreux couples, sont souvent en désaccord.

Chères lectrices, chers lecteurs, vous allez maintenant penser que je n'ai pas tout compris, que ce couple n'est que le fruit de l'habitude, dans le langage familier, de personnifier deux aspects d'un seul et même individu. En réalité, «mon corps et moi» ne sommes pas deux êtres distincts, mais bien un seul.

J'entends bien, mais je doute que l'on puisse balayer si facilement la question. Il apparaît en effet que nous pensons comme nous parlons et que nous agissons comme nous parlons et pensons.* Cela vaut d'ailleurs aussi pour les actes médicaux. Notre corps est examiné, ausculté et réparé jusqu'à plus soif grâce à l'imagerie, aux résultats d'analyse, aux biopsies, aux endoscopies et à la chirurgie. Les problèmes surviennent lorsque, malgré des recherches intensives, on ne trouve pas le défaut ou lorsque celui-ci ne peut pas être réparé avec les ressources disponibles. La pratique médicale consiste à soigner mon corps, je suis quant à moi exclu de ce processus. Ce n'est d'ailleurs pas nouveau. Platon subdivisait déjà l'être humain en multiples éléments et parlait, par exemple, du corps comme d'une prison pour notre âme [1]. La quasi-totalité des cultures distinguent notamment le corps et l'âme. Chez les Egyptiens de l'Antiquité, cette dernière comptait ellemême trois composantes, appelées $\mathrm{Ka}$, Ba et Akh.

A l'opposé, il y a les moments où nous nous sentons profondément en communion avec nous-mêmes, où nous savons avec une certitude absolue que les sages d'Orient ont raison lorsqu'ils nous permettent de faire l'expérience, lors de la méditation et des exercices de yoga, que nous ne faisons qu'un avec toutes nos composantes. Et si cette expérience d'indivisibilité ne nous trompe pas, le discours sur «mon corps» employé en médecine (et ailleurs) ne serait rien d'autre qu'une forme de détachement de nous-mêmes. Nous perdons notre unité et sommes soudain confrontés à un autre: le corps. Et cela va plus loin encore: dès lors que nous sommes étrangers à nous-mêmes, nous avons du mal à percevoir notre environnement tel qu'il est, nous nous dissocions de nous-mêmes mais aussi de ce qui nous entoure. Peut-être la destruction de notre environnement vient-elle de là, de la dislocation de notre caractère uni et indivisible.

Vous allez me dire: "Ça suffit! Vous vous égarez complètement, vous divaguez au sujet de liens de cause à effet à l'échelle planétaire, vous perdez tout contact avec la réalité!» C'est vrai. Revenons-en à ce qui est pratique et concret, comme il sied à des professionnels de la santé, et penchons-nous à nouveau sur les habitudes du langage courant. Au lieu de: «J'ai poussé mon corps au-delà de ses limites, il a fini par se rebeller, par m’asséner 'Ça suffit!' et par me jeter au lit», pourquoi ne pas simplement dire: «Je me suis tellement surmené que je suis tombé malade»? Et au lieu de: «Au fil des ans, j’ai appris à écouter mon corps et à lui faire confiance», simplement "Au fil des ans, j'ai appris à m'écouter et à me faire confiance»?

Ce sont des petits pas, faciles à appliquer au quotidien. Pratiqués sur la durée, ils pourraient avoir des effets inattendus.

* Voir l'aphorisme de Charles Reade (écrivain anglais, 1814-1884):

"Prenez garde à vos pensées car elles deviendront vos mots.

Prenez garde à vos mots car ils deviendront vos actes. Prenez garde à vos actes car ils deviendront vos habitudes.

Prenez garde à vos habitudes car elles deviendront votre caractère.

Et prenez garde à votre caractère car il deviendra votre destinée."

\section{Référence}

1 Platon: Phédon (82 d) 